\title{
La chanson néo-crétoise : ressourcement et confluences
}

\section{Vassiliki Coavoux}

\section{(2) OpenEdition}

Journals

Édition électronique

URL : http://journals.openedition.org/transtexts/217

DOI : $10.4000 /$ transtexts. 217

ISSN : 2105-2549

Éditeur

Gregory B. Lee

Édition imprimée

Date de publication : 1 janvier 2008

Pagination : 49-59

ISSN : 1771-2084

\section{Référence électronique}

Vassiliki Coavoux, «La chanson néo-crétoise : ressourcement et confluences », Transtext(e)s

Transcultures 跨文本跨文化 [En ligne], Hors série | 2008, mis en ligne le 14 septembre 2009, consulté le 19 avril 2019. URL : http://journals.openedition.org/transtexts/217 ; DOI : 10.4000/transtexts.217 
Poésie et Insularité - Poetry and Insularity

\title{
La chanson néo-crétoise : ressourcement et confluences
}

\author{
VASSILIKI COAVOUX \\ Jean Moulin University - Lyon
}

For the last thirty years in Crete there has developed, alongside poetry and the still thriving oral tradition of Cretan songs, a new «neo-Cretan » trend of popular and traditional song inspiration that nevertheless reaches beyond tradition in both music and lyrics. The body of songs presented here, the Haïnides, is probably the most representative of this trend. This article aims at analysing the lyrics of their first six albums in comparison with poetry of the oral tradition in order to better understand the neo-Cretan movement.

Nous proposons ici un rapide survol de l'évolution de la poésie et de la chanson crétoises de tradition orale, depuis1950 jusqu'à nos jours, et plus particulièrement à travers le mouvement néo-crétois tel qu'il s'est développé depuis les années $1980 .{ }^{1}$ Notre but est de montrer comment le chant et la musique ont réagi devant les mutations de la société après la guerre en traversant tour à tour une phase de recul,

\footnotetext{
${ }^{1}$ La poésie crétoise de tradition orale fut recueillie et éditée, tout comme l'ensemble de la poésie populaire grecque, à partir du milieu du XIXe siècle. Voici les principaux recueils : Apostolakis Stamatis, Rizitika: les chants populaires crétois, Athènes, Gnossi, 1993 ; Baud-Bovy Samuel, Chansons populaires de Crète occidentale, Genève, Minkoff, 1972 ; Détorakis Théocharis, Chants populaires crétois inédits, Héraclion, 1976 ; Kriaris Aristide, Chants populaires crétois, Athènes-La Canée, 1920 ; Lioudaki Maria, Mandinadès, Gnosseis, Athènes, 1936 ; Papagrigorakis Idoménée, Les chants Rizitika crétois, La Canée, 1957 ; Vavoulès Pandèlis, Le chanteur crétois, La Canée, Société ethnographique et archéologique, 2003.
} 
puis une de retour aux sources, avant l'arrivée du mouvement que nous appelons néo-crétois.

Après un bref exposé historique concernant la poésie populaire, nous nous intéresserons à un groupe de musiciens et chanteurs néo-crétois, les Haïnidès, qui constituent un bon exemple de ce mouvement.

Jusqu'aux années 1950, la poésie de tradition orale avait très peu évolué car la Crète était restée isolée et avait longtemps vécu à l'écart de la modernisation, comme beaucoup d'autres îles et régions du monde rural grec. Nous n'irons pas jusqu'à affirmer que la modernisation en matière musicale ne l'avait pas touchée : dans le milieu des années 1950, par exemple, dans mon village natal, lors des fêtes, on jouait et chantait valses et tangos après les airs traditionnels, mais tard dans la soirée, comme si l'on avait un peu honte de le faire. Et dix ans plus tard, les chansons de Mikis Théodorakis étaient elles aussi arrivées jusqu'à mon village.

A cette date, la Grèce se trouvait déjà parvenue à une phase moderne et posttraditionnelle en ce qui concerne la musique et les textes des chansons populaires populaires au sens large du terme -, grâce à Manos Hadjidakis et à Mikis Théodorakis, qui avaient rénové les deux, en puisant en particulier dans la tradition musicale du rébétiko, ainsi qu'en utilisant des textes de nos poètes contemporains ou en mettant en musique des textes de grande valeur. ${ }^{2}$

Cependant, la musique folklorique traditionnelle et la poésie chantée se maintenaient au sein du monde rural. En Crète, les Rizitika et les mandinadès constituaient, comme dans le passé, l'essentiel du répertoire. Les Rizitika sont des poèmes souvent narratifs et plus rarement lyriques ou descriptifs, chantés autour de la table et lors des déplacements, pendant les noces, les fiançailles ou les baptêmes. Les mandinadès sont quant à elles des distiques autonomes qui accompagnent les danses traditionnelles.

Mais la modernité n'était pas loin. Dans les années 1950-1960, la radio pénètre dans les maisons et le disque en vinyle, avant la cassette, devient un produit presque courant. Ils conduisent à une grande uniformisation des goûts et, surtout, brisent l'enclavement des régions rurales qui entrent ainsi en contact avec des musiques et des chants jusqu'alors inconnus. La décennie suivante, 1960-1970, est celle de

\footnotetext{
${ }^{2}$ Le terme "populaire» traduit en français deux mots du grec qui désignent deux catégories très différentes de chansons : les démotiques, qui correspondent au folklore rural et dont certaines remontent au Moyen Age, et les laïka qui datent du XIXe ou du XXe siècle et correspondent au « folklore » urbain. Une partie de ces derniers est connue sous le nom de rébétika.
} 
l'émigration massive des Grecs et des Crétois vers l'Allemagne, l'Australie et d'autres pays, ainsi que de l'exode rural. Pratiquement au même moment commence le développement du tourisme de masse. La société traditionnelle et sa culture sont ébranlées. A la campagne, les villages se dépeuplent et les traditions s'y maintiennent difficilement. Dans les villes et les lieux envahis par les touristes voient le jour des chansons et des musiques «pour touristes». C'est pour la chanson traditionnelle une période de stagnation, probablement même de recul.

Au début des années 1980, on observe une période de désintérêt de la part des jeunes, qui correspond grosso modo à la première génération après l'exode rural et à l'avènement du tourisme de masse. Les enfants de paysans nés à la ville n'avaient pas été nourris de musique traditionnelle et, dans les villes et les bourgades, peutêtre n'était-il pas valorisant à leurs yeux de chanter des chants de paysans.

Cette période reste toutefois de courte durée. Sous la pression familiale ou de leur propre initiative, ces nouveaux citadins envahissent très vite les écoles de danses folkloriques, les cours de lyre, de luth et autres instruments traditionnels, ou encore les chorales. Le retour vers la tradition est spectaculaire. Il semble certes quelque peu artificiel et sa vigueur telle que c'en est presque trop. En même temps, les enregistrements de cassettes se multiplient de façon tout aussi spectaculaire. Des musiciens et chanteurs jusqu'alors inconnus connaissent la gloire, très souvent après un court séjour en Australie, en Allemagne ou ailleurs, au sein des communautés de Crétois expatriés (à ce propos, le rôle de la diaspora grecque pour le maintien des traditions serait intéressant à étudier). Les antennes des radios locales et nationales diffusent également des émissions de musique et de chants folkloriques. A remarquer qu'à cette époque, l'accent dialectal crétois est utilisé à outrance par les chanteurs et par les présentateurs.

Ce retour à la fois quelque peu artificiel et spectaculaire vers la tradition était dans une certaine mesure dérangeant, mais il avait le mérite d'assurer la continuité et, à ce titre, méritait le respect.

Les deux dernières étapes de l'évolution du chant et de la musique insulaires correspondent également à la période d'activité et de gloire de Nikos Xylouris et de ses disciples. Ce musicien et chanteur exceptionnel, après une courte carrière à Héraclion, se rend à Athènes dès 1969 et fait sortir de Crète la musique et les chansons crétois qu'il fait connaître au grand public grec. Il collabore avec de grands compositeurs tels que Marcopoulos, Léondis, Xarchacos, etc., et son répertoire dépasse vite la seule chanson crétoise. Le timbre de sa voix, son accent 
caractéristique, le dialecte de son village deviennent familiers aux Athéniens et aux autres Grecs dans des textes et des musiques qui pourtant ne sont pas toujours crétois. La connotation « musique folklorique » s'atténue lorsque Xylouris chante, et ce malgré les marques dialectales très ostensibles de sa voix ou de sa langue. Ce phénomène est comparable à ce qui s'était passé dans les années 1950 avec le rébético et la voix de Bithicotsis. La musique traditionnelle, grâce à un concours de circonstances et à des hommes de grand talent, est " modernisée », mise au goût du jour et revalorisée, sans pour autant perdre son caractère.

Ce processus de modernisation de la musique et de la chanson crétoises amorcé avec Xylouris va se poursuivre après sa mort prématurée en 1980 (il avait alors 44 ans). Après lui, des gens de sa famille, de son village, ou de sa région, ou encore d'autres Crétois, ses disciples en quelque sorte, inaugurent en Crète un courant que l'on peut appeler néo-crétois (le terme local utilisé est "critogénis moussiki »). Parmi les noms les plus connus, nous pouvons citer ceux de Haralambos Garganourakis, Psarandonis, Loudovicos d'Anogia, l'Irlandais, Crétois d'adoption, Ross Daly, ainsi que notre groupe, les Haïnidès. Mais de nombreux jeunes musiciens et chanteurs de grand talent, amateurs ou professionnels, inconnus du grand public, suivent ce courant en Crète, et nous en avons personnellement rencontré quelques-uns. Ils partent de la tradition, conservent certains de ses éléments mais la dépassent en sortant des modèles imposés par elle.

Le groupe Haïnidès est très représentatif du courant néo-crétois. Le mot Haïnis est d'origine turque et signifie en dialecte crétois le hors-la-loi, le révolté, l'homme « en cavale », l'insoumis. L'ensemble a été créé en 1990 à Héraclion par des jeunes gens originaires de la région, presque tous nés vers 1970. Ce n'est pas un groupe figé : hormis le poète-compositeur et musicien Dimitris Apostolakis et un noyau stable de quatre ou cinq personnes dont une femme, des artistes invités, des amis et membres temporaires participent aux enregistrements et aux concerts. ${ }^{3}$

Outre les instruments traditionnels crétois - à savoir principalement la lyre, le violon et le luth et, plus rarement, la mandoline et la mandole -, ils utilisent également le saz, le 'oud, le bendir, la derbouka, mais aussi le violoncelle, la guitare, la flûte, des instruments du rébétiko tels que le dzouras, le tambouras, ou des instruments anciens oubliés et remis au goût du jour par le mouvement néo-crétois comme le boulgari, etc. La réutilisation de ces instruments anciens est l'une des contributions importantes du mouvement au maintien de la tradition.

\footnotetext{
${ }_{3}$ Parmi les membres permanents, nous pouvons citer les noms de Maria Koti, Michalis Stavrakakis, Dimitris Zacharioudakis, Kallia Spyridaki, Kostis Avyssinos, etc.
} 
N'étant ni musicienne ni spécialiste de la musique, je peux seulement affirmer que sur ce plan le groupe est resté assez fidèle à la tradition de la Crète centrale. Certaines chansons la respectent absolument; pour d'autres, la mélodie est beaucoup plus douce et plus lente que celles de la musique traditionnelle et

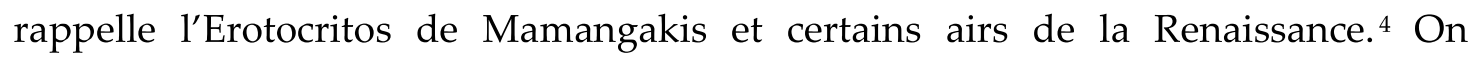
observe aussi des mélanges : des mandinadès crétoises sont chantées sur des airs non traditionnels et, à l'inverse, des textes très éloignés de la tradition sur des airs traditionnels. Mais le fait est que le public reconnaît en eux des musiciens crétois et accourt à leurs concerts.

De 1991 à 2002, ils ont enregistré six albums : Haïnidès (1991, neuf poèmes), Le monde et le rêve ne font qu'un (1993, huit poèmes), A contre-courant (1994, onze poèmes), Le grand voyage (1997, treize poèmes), Le prince aux pieds nus (2000, vingt poèmes), Bulletin d'informations (2003, trois poèmes). ${ }^{5}$

Nous nous proposons d'examiner les convergences et les divergences que ces textes présentent par rapport à la poésie populaire crétoise chantée, de les classer et d'essayer de comprendre leur fonctionnement. Rappelons que la poésie de tradition orale est à l'origine l'œuvre d'un individu, reconnue et adoptée par un groupe dont elle exprime les sentiments et respecte les valeurs esthétiques. Le temps efface ensuite le nom du poète et c'est dans ce sens que l'on utilise le terme «création collective $»{ }^{6}$

Parmi les soixante-quatre poèmes du corpus, dix sont des textes traditionnels, dont cinq repris tels quels, et cinq sont des adaptations. L'un est un poème de Costas Caryotakis; quarante-quatre ont été écrits par Dimitris Apostolakis, membre du groupe, et le reste par trois autres de ses membres. Notre classement respectera les deux grandes catégories traditionnelles, mandinadès et Rizitika, auxquelles s'ajoutent les poèmes de création originale. Le poème de Caryotakis ne sera pas classé.

\footnotetext{
${ }^{4}$ Le compositeur Nikos Mamangakis, né à Réthymnon en 1929, a composé des œuvres s'inspirant à la fois de la musique occidentale de la Renaissance, de la musique traditionnelle crétoise et de musique moderne. Son Erotokritos, inspiré du roman crétois du XVIIe siècle, date des années 1960.

5 Un nouvel double album de trente-six textes, qui porte le titre L'enchanteur, vient de paraître en 2005.

6 Sur la question de la création " collective », voir le livre d’Eratosthénis Kapsoménos, La poésie populaire grecque, Réthymnon, 1978 (notes à l'usage des étudiants, en grec), p 44-46.
} 


\section{1) Les mandinadès}

Ce nom désigne les distiques rimés, composés de vers iambiques de quinze syllabes avec césure fixe après la huitième syllabe. ${ }^{7}$ Les distiques existaient déjà aux $\mathrm{XV}^{\mathrm{e}}$ XVIe siècles comme le prouvent les manuscrits de Vienne édités par Emile Legrand en1874 et Hubert Pernot en 1913. ${ }^{8}$ Jusqu'à nos jours, elles accompagnent essentiellement les danses. Les chanteurs connaissent un très large répertoire qui leur sert de canevas à l'improvisation, et adaptent souvent leurs textes aux circonstances. Encore aujourd'hui, elles représentent le genre le plus populaire, le plus vivant et le plus prolifique de la poésie traditionnelle en Crète.

Dans notre corpus, les mandinadès sont utilisées non pas isolément et de façon autonome, comme c'est le cas dans la poésie populaire, mais de manière plus sophistiquée, par groupes de deux, trois ou quatre distiques qui forment un court texte doté d'un titre, ou intégrées dans des textes plus longs. Pour le premier cas de figure, le plus proche de la tradition, nous avons compté trente-trois distiques ainsi répartis :

Album 1 : trois groupes de deux mandinadès

Album 2: un groupe de trois

Album 3 : un groupe de quatre, deux groupes de deux

Album 4 : un groupe de deux, un de trois, un de quatre

Album 5 : deux groupes de deux, un de trois

Ainsi, dans l'album Haïnidès, les poèmes Le tambouras de Stavros, Cantate, L'insoumis sont composés chacun de deux distiques. Pour le deuxième cas de figure : lorsque les mandinadès sont intégrées à des poèmes plus longs et beaucoup plus complexes, on s'éloigne de la tradition. Toujours dans le premier album (Haïnides), le poème Délire est composé de trois unités, trois strophes, comprenant chacune deux vers oxytons de dix-huit syllabes, suivis de trois mandinadès. Ou encore dans l'album $\mathrm{n}^{\circ}$, Le prince aux pieds nus, le poème L'assassin est composé d'une longue introduction en vers libre, suivie de trois mandinadès.

\footnotetext{
7 Le terme «iambique », emprunté à la métrique du grec ancien, désigne un groupe de deux syllabes, dont la première ne porte pas d'accent et la deuxième est accentuée. La prosodie a en effet disparu en grec moderne et le rythme est obtenu par la succession régulière de syllabes accentuées ou non accentuées.

${ }^{8}$ Le premier éditeur, W. Wagner, avait formulé l'hypothèse que ces poèmes provenaient de l'île de Rhodes. Voir W. Wagner, Das ABC der Liebe, Leipzig, 1879, et D. C. Hesseling et H. Pernot, Chansons d'amour, Paris-Athènes, 1913.
} 


\section{2) Les poèmes inspirés de la tradition}

L'autre grande catégorie de poèmes crétois est celle des Rizitika. Le terme signifie littéralement «chants du Piémont », à savoir des villages situés sur les contreforts des Montagnes Blanches, au nord-ouest de la Crète. En réalité, dans toute l'île, le terme est générique et désigne toutes les chansons narratives ou évoquant une histoire, ainsi que les chansons dites « de la table». Les plus anciens textes connus sont ceux qui ont été conservés dans des manuscrits du XVIIe siècle des monastères d'Iviron et Xyropotamou, au Mont Athos. ${ }^{9}$ Contrairement aux mandinadès, ces textes, dans leurs différentes versions (ou variantes), sont figés et, à quelques mots près, on ne peut pas les modifier ou les adapter sans altérer leur caractère.

Toutefois, en écoutant ou en lisant les textes des Haïnidès, on ne peut que constater leur parenté étroite avec les poèmes narratifs crétois et plus précisément avec les Paralogues (les Ballades). Cette parenté n'est pas formelle. Les Rizitika utilisent le même vers iambique de quinze syllabes que les mandinadès et sont presque toujours narratifs. Les poèmes des Haïnides suggèrent seulement une histoire et leur vers est rarement iambique mais de nombreux thèmes et motifs proviennent de la poésie traditionnelle.

Nous pouvons classer dans un premier groupe tous les poèmes du corpus dont le sujet rappelle des textes connus de la tradition orale.

Par exemple, dans l'album ${ }^{\circ} 1$, le texte $n^{\circ} 8$, La mort $d u$ Jeune homme, dont le titre est emprunté à Costis Palamas, le narrateur-poète raconte qu'il a blessé un jeune homme à la guerre .Celui-ci, à l' agonie, souhaite se transformer en arbre après sa mort, tout près d'une fontaine, afin de contempler les belles qui viendront chercher de l'eau et de voir sa bien-aimée. Le motif est emprunté aux Klephtika. ${ }^{10}$

Dans l'album n`2, La cage en or, dont le titre et le thème sont empruntés à la tradition, est l'histoire d'un faucon, capturé très jeune et élevé avec beaucoup de soins dans une cage en or, qui se tue en essayant de s'échapper de sa prison. ${ }^{11}$

Dans le même album, Le Géant, L'homme de quarante coudées, aussi bien le titre que le contenu du poème évoquent les variantes crétoises de la légende de Dighénis. ${ }^{12}$

\footnotetext{
${ }^{9}$ Le manuscrit a été édité par Bertrand Bouvier, Chants populaires d'un manuscrit $d u$ Monastère d'Iviron, Athènes, 1960.

${ }^{10}$ Littéralement «Chants des brigands » datant des XVIII et XIXe siècles, qui célèbrent les exploits et la vie des Grecs qui se battaient contre l'occupant ottoman.

11 Voir le recueil de A. Kriaris, Chants populaires crétois, La Canée-Athènes, 1909 (en grec), p. 346.
} 
Mais dans le texte des Haïnides, le héros, égaré dans l'époque moderne, privé de monstres, de fauves et d'exploits à accomplir, se suicide. Il n'y a plus de place pour les héros.

Dans l'album $\mathrm{n}^{\circ} 3$, le texte Ali, le corsaire sarrasin est inspiré des nombreuses légendes populaires liées à l'action des Barbaresques dans la région. ${ }^{13}$ Ali, enlevé alors qu'il était encore enfant, devient corsaire. Jeune homme, il tombe amoureux d'une femme (probablement crétoise) et, pour elle, laisse repartir sa galère et reste à terre. Plus tard, il reprend la mer, mais ses vieux jours venus, il retourne sur la terre où il avait aimé.

Dans l'album $\mathrm{n}^{\circ} 4$, le poème La Néréide évoque les légendes crétoises de ces femmes dévoreuses d'hommes, sur le thème de l'amour malheureux. Mais nous voulons croire qu'implicitement, il s'agit plutôt de la légende concernant l'apprentissage de la lyre que seules ces créatures peuvent enseigner aux apprentis musiciens.

Dans l'album $\mathrm{n}^{\circ} 2$, le texte intitulé La jeune Catherine rappelle beaucoup la ballade populaire du même nom. Il évoque la mort prématurée d'une jeune femme très belle, sans plus de précisions. Il s'agit probablement de souvenirs de deux poèmes traditionnels, celui de l'épouse infidèle et des nombreuses variantes de La mal mariée. ${ }^{14}$

Il existe enfin un groupe de textes, parfois adaptations de poèmes traditionnels, $\mathrm{s}$ 'inspirant librement $\mathrm{du}$ thème très répandu de l'expatriation, comme par exemple Évocations dans l'album n²1, ou encore Sourmélim dans le n³, La jeune Elpida dans le même album, Bateau petit bateau dans le ${ }^{\circ} 4$.

Pour tous les poèmes de cette catégorie, il s'agit toujours d'inspiration libre : les textes traditionnels fournissent seulement les thèmes ou les motifs qui sont traités et développés librement sur le plan formel.

\footnotetext{
12 Personnage légendaire de l'épopée du Moyen Age et héros de nombreux poèmes populaires dans toute la Grèce.

13 A ce sujet, voir notre article «Le thème de la mer dans la poésie populaire crétoise » in May Chehab, Yannis Ioannou, Françoise Métral, Méditerranée, ruptures et continuités, Maison de l'Orient méditerranéen, Lyon, 2003, p. 67-72.

14 Voir Détorakis, p. 94, et Apostolakis, p. 214-219.
} 


\section{3) Les poèmes de création originale}

Tous les autres poèmes sont des créations personnelles, sans grands rapports avec la tradition. L'amour en est un des thèmes majeurs. Le poète s'adresse souvent à la bien-aimée présente ou absente. Une femme sur le point de partir hante nombre de ces textes. L'autre thème principal est la tristesse et la mélancolie liées à l'amour, car il n'est que rarement question des joies qui l'accompagnent. L'amour est ici synonyme de chagrins, de souffrances et de souvenirs qui éveillent la tristesse. Citons quelques titres évocateurs: Ne reviens pas, J'avais jadis un amour, ou encore Étoile du sud qui parle de trahison.

Quelques poèmes ont pour sujet l'amitié, dans le passé lointain de l'enfance ou de l'adolescence, comme Le cabaret ou Nikitas, moi-même et Stélis dans l'album n 1 . On relève enfin des textes plus lyriques, empreints également d'une douce mélancolie, de tristesse contenue et de pessimisme. Le fait qu'un poème de Costas Caryotakis, Même si mon rêve s'est effacé telle une ombre, soit présent dans l'album $\mathrm{n}^{\circ} 4$, et dédié à sa mémoire, révèle la présence de références littéraires, qui sont en effet manifestes dans d'autres compositions.

Nous avons déjà mentionné le titre emprunté à la nouvelle de Palamas. En ce qui concerne Caryotakis, outre le poème mentionné plus haut, nous pensons que la mélancolie, le pessimisme, la présence de la mort, relèvent de son héritage. Par ailleurs, deux textes au moins sont inspirés de Xanthoula, le poème de D. Solomos, en même temps que de poèmes traditionnels sur l'expatriation. Il s'agit de Sourmélim (album $\mathrm{n}^{\circ}$ ) et de Bateau petit bateau (album $\mathrm{n}^{\circ} 4$ ). La mélodie ancienne, dans $l^{\prime}$ album $n^{\circ} 1$, qui évoque une séparation et une femme aimée partie un soir pluvieux d'automne, comporte manifestement des souvenirs de l'Hélène d'O. Elytis. Dans la chanson Le mariage (album $\mathrm{n}^{\circ} 4$ ), le rythme et le vers rappellent Eroticos Logos de G. Séféris (la troisième partie, avec l'alternance d'un vers paroxyton de quinze syllabes et d'un vers oxyton de quatorze, avec rime croisée). Dans sa première strophe nous retrouvons le motif des amoureux qui se fiancent avec des branches coupées au bord du chemin, comme dans le poème du XVIe siécle intitulé La jeune bergère. D'autres emprunts, similitudes ou clins d'œil nous ont probablement échappé. ${ }^{15}$

En ce qui concerne la forme, cette catégorie de poèmes utilise une métrique et une versification très élaborées et complexes, bien éloignées de la poésie traditionnelle.

15 Odysseus Elytis, Orientations, Ikaros, Athènes, 1987 (11e éd.), p. 75 . Georges Séféris, Poèmes, Ikaros, Athènes, 1967, p. 34-35. La jeune bergère, poème du XVIe siècle, d'auteur inconnu, édité par Stylianos Alexiou, Ermis, 1971, p. 68. 
Faisons maintenant un bilan rapide. Sur les soixante-quatre poèmes du corpus, douze sont composés de mandinadès et dans neuf autres des mandinadès sont intégrées à des compositions élaborées. Sept sont, par leur inspiration, proches des Rizitika. Tous les autres sont des textes originaux.

Comment expliquer alors l'adhésion du public crétois et le succès du groupe? Quelle alchimie - outre la présence de la musique, qui joue indéniablement un rôle déterminant - fait que le public reconnaît comme siens ces textes souvent bien éloignés de l'esthétique de la chanson populaire ? Trois caractéristiques semblent, à notre avis, servir d'identifiants et permettre au public de reconnaître sa culture, sa langue et l'esthétique traditionnelle.

Tout d'abord, la présence du dialecte crétois. Avec parcimonie et art, les poèmes sont parsemés d'éléments dialectaux qui sont plus au moins noyés dans le reste et passent inaperçus à la simple lecture, tout en étant parfaitement audibles lorsque ces poèmes sont chantés. Une certaine emphase de l'accent dialectal que l'on peut remarquer vient peut-être remédier à l'absence quantitative de ces marqueurs. Pour en donner une idée, nous avons relevé trente et une occurrences d'éléments dialectaux dans le premier album, tous niveaux confondus, une dizaine seulement dans le quatrième.

Vient ensuite la présence des mandinadès. Etant donné que le genre est encore très vivant et que les musiciens et chanteurs populaires en composent toujours, elles trouvent parfaitement leur place ici. Disséminées habilement dans tous les albums, elles ponctuent et rythment les autres textes grâce à la musique de danse et aux paroles issues de la tradition ou très proches d'elle. En même temps, elles servent en quelque sorte de signe de ralliement : leur présence est la preuve qu'il s'agit bien de chants crétois, même lorsqu'elles sont intégrées dans des textes complexes.

On relève enfin la présence de nombreuses références à la culture locale, elles aussi dispersées à très petites doses dans les textes. Il s'agit d'abord de références historiques allant de l'époque médiévale et de l'occupation vénitienne à l'occupation ottomane et aux souvenirs de la catastrophe d'Asie Mineure. A une occurrence près, l'histoire et la mythologie antiques sont absentes. On note ensuite des clins d'œil à la vie quotidienne : la tsikoudia, eau de vie locale que l'on boit accompagnée de noix, les noms dialectaux des instruments de musique, les plumes de faucon utilisées comme plectre, la patania, couverture souvent rouge brodée au métier à tisser, etc. On trouve aussi un virelangue, sans oublier des allusions à quelques légendes locales et contes de grand-mère. 
Ainsi, les éléments empruntés à la tradition sont loin d'être majoritaires et prédominants. Ils sont utilisés à dessein pour assurer la filiation, et les Haïnidès comme tous les néo-Crétois n'en veulent pas davantage. La chanson néo-crétoise ne saurait exister sans cette filiation. Mais ce à quoi aspirent ces jeunes gens (et ils le disent clairement dans le premier texte de leur premier album, qui porte le titre Les contes de grand-mère), ce n'est pas de rester simplement des chanteurs et musiciens traditionnels comme tant d'autres. Ils cherchent un mode d'expression qui, partant de la tradition et s'inspirant d'elle, va bien plus loin. Ils acceptent l'héritage du passé, se tournent vers lui avec respect et admiration, mais aussi avec la volonté de s'en servir, de s'en inspirer et de créer quelque chose de nouveau. Leur aspiration, si l'on en juge par leur succès, correspond et répond à une demande du public, jeune et moins jeune. Dans quelques décennies (ou quelques siècles), leurs chansons feront peut-être à leur tour partie de la tradition, lorsque les noms et les circonstances de leur création seront oubliés. Qui sait? 